\title{
Best MRI sequences for identifying axillary lymph node markers in patients with metastatic breast cancer: an inter-reader observational study
}

\author{
Naziya Samreen ${ }^{1}$, Asha A. Bhatt ${ }^{2 *}$, Kalie Adler ${ }^{3}$, Shannon Zingula ${ }^{2}$ and Katrina N. Glazebrook ${ }^{2}$
}

\begin{abstract}
Background: We assessed confidence in visualization of markers within metastatic axillary lymph nodes (LNs) on magnetic resonance imaging (MRI), which were placed post-ultrasound (US)-guided biopsy.

Methods: A retrospective review was performed on 55 MRI cases between May 2015 and October 2017. Twentytwo MRIs were performed before neoadjuvant therapy, and 33 MRIs were after its initiation. There were 34/55 HydroMARK $^{\oplus}, 10 / 55$ Tumark $^{\oplus}$, and 11/55 other marker types. Time interval between marker placement and MRI examination was $103 \pm 81$ days (mean \pm standard deviation). Three readers with 1-30 years of experience independently assessed four axial sequences: unenhanced fat-suppressed three-dimensional T1-weighted spoiled gradient-recalled (SPGR), first contrast-enhanced fat-suppressed SPGR, T2-weighted water-only fast spin-echo (T2WO), and T2-weighted fat-only fast-spin-echo (T2-FO).

Results: Markers were $5.2 \times$ more likely to be visualized on T2-WO than on unenhanced images $(p=<0.001)$, and $3.3 \times$ more likely to be visualized on contrast-enhanced than on unenhanced sequences $(p=0.009)$. HydroMARK markers demonstrated a $3 \times$ more likelihood of being visualized than Tumark $(p=0.003)$. Markers were $8.4 \times$ more likely to be visualized within morphologically abnormal LNs $(p<0.001)$. After 250 days post-placement, confidence in marker brightness of HydroMARK markers on T2-WO images was less than 50\% $(p<0.001)$. Inter-rater agreement was excellent for T2-WO and contrast-enhanced SPGR, good for unenhanced SPGR, and poor for T2-FO images.

Conclusion: T2-WO and contrast-enhanced images should be used for marker identification. HydroMARK was the best visualized marker. Markers were easier to identify when placed in abnormal LNs. The visibility of HydroMARK markers was reduced with time.
\end{abstract}

Keywords: Axilla, Breast neoplasms, Lymph nodes, Magnetic resonance imaging, Surgical instruments

\section{Key points}

- T2-weighted water-only images have a higher probability of allowing marker identification compared to other imaging sequences.

\footnotetext{
* Correspondence: bhatt.asha@mayo.edu

²Department of Radiology, Mayo Clinic, 200 1st street SW, Rochester, MN 55905, USA

Full list of author information is available at the end of the article
}

- HydroMARK markers are more easily visualized on MRI compared to Tumark markers.

- Markers are easier to identify when the lymph node demonstrates abnormal morphology.

\section{Background}

According to the National Comprehensive Cancer Network guidelines, systemic imaging should be considered in patients who are clinically stage IIB with advanced axillary disease, stage III, locally advanced, and in those

\section{Springer Open}

() The Author(s). 2020 Open Access This article is licensed under a Creative Commons Attribution 4.0 International License, which permits use, sharing, adaptation, distribution and reproduction in any medium or format, as long as you give appropriate credit to the original author(s) and the source, provide a link to the Creative Commons licence, and indicate if changes were made. The images or other third party material in this article are included in the article's Creative Commons licence, unless indicated otherwise in a credit line to the material. If material is not included in the article's Creative Commons licence and your intended use is not permitted by statutory regulation or exceeds the permitted use, you will need to obtain permission directly from the copyright holder. To view a copy of this licence, visit http://creativecommons.org/licenses/by/4.0/. 
with inflammatory breast cancer [1]. Magnetic resonance imaging (MRI) can help in the assessment of disease extent, including axillary lymphadenopathy, and change in tumor burden post-neoadjuvant therapy (NAT) [1, 2]. Assessment of breast cancer axillary lymph node (LN) metastases is critical for staging and surgical planning [3-7].

In patients with breast cancer who have suspicious axillary LNs, a marker is often placed within the LN at time of biopsy. Prior studies have established the importance of marker placement in the biopsy proven LN at diagnosis [8-12]. A marker is placed within suspicious or cytology/pathology proven metastatic LNs to facilitate localization and subsequent surgical removal. Hence, when these patients undergo MRI for staging or for assessment of response to NAT, the marker can be visualized on MRI. Thus, it is important to know which MRI sequences best demonstrate the marker because this helps identify the biopsy proven metastatic LN. Additionally, if the metastatic LN decreases in size on the post-NAT MRI, similar landmarks can be used to identify the marked metastatic $\mathrm{LN}$ to evaluate response to treatment.

The purpose of this study is to assess confidence in axillary LN marker visualization on four different MRI sequences to determine which sequence is the most useful in identification of the marker to help monitor response to NAT.

\section{Methods}

After Institutional Review Board approval (Mayo Clinic Rochester, MN, October 17, 2016, ID 16-008036), a retrospective review was performed of all patients who underwent axillary LN biopsy with marker placement and subsequent breast MRI between May 2015 and October 2017 (29 months).

The two main markers used at our institution for axillary LN biopsies include the 18 gauge HydroMARK ${ }^{\circ}$ markers (Mammotome, Cincinnati, OH) and Tumark ${ }^{\circ}$ markers (Hologic, Bedford, MA). A HydroMARK marker has a biodegradable hydrogel polymer containing a central permanent metal marker. The hydrogel polymer immediately expands with deployment, containing > 90\% water as hydration, which can last up to 12 months [13]. A Tumark marker consists of two parts: a metallic core made of titanium or stainless steel and a bioabsorbable suture-like netting that surrounds the metal [14].

All MRIs were performed at 1.5 Tesla (NY scanner, General Electric, Boston, MA) using a 7-channel dedicated bilateral breast coil. Axial, T1-weighted fatsuppressed three-dimensional spoiled gradient-recalled (SPGR) images were acquired before intravenous injection of gadolinium-based contrast agent and after intravenous injection of gadolinium-based contrast agent (Gadavist, $0.1 \mathrm{mmol} / \mathrm{kg}$ ) at $1-1.5 \mathrm{~min}, 2-3 \mathrm{~min}$, and about 7-8 min using a field of view of $28-40 \mathrm{~cm}$, slice thickness $1.8-2.0 \mathrm{~mm}$, in-plane resolution $\leq 1.0$ $\mathrm{mm}^{2}$, repetition time $5.2 \mathrm{~ms}$, echo time $2.5 \mathrm{~ms}$, flip angle $10^{\circ}$, bandwidth $62.50 \mathrm{kHz}$, and phase direction left-to-right. Axial T2-weighted water-only (T2-WO) and fat-only (T2-FO) images were acquired using a fast spin-echo acquisition using a modified 3-point Dixon method for fat suppression (iterative decomposition of water and fat with echo asymmetry and least-squares estimation, IDEAL), field of view 28-40 $\mathrm{cm}$, slice thickness $4.0 \mathrm{~mm}$ with $1.0 \mathrm{~mm}$ spacing, repetition time $4,505 \mathrm{~ms}$, echo time $102 \mathrm{~ms}$, echo-train length 14 , matrix $320 \times 224$, number of excitations 2.0 , and bandwidth $50.0 \mathrm{kHz}$.

Three readers with 1-30 year experience in breast MRI interpretation independently assessed for visualization of markers on the four sequences in the following order: (1) T2-WO, (2) T2-FO, (3) unenhanced SPGR, and (4) first contrast-enhanced SPGR. Readers were blinded to results from other readers and to the type of marker placed. An assessment was made by each reader whether the marker was visualized or not. Visualization of marker meant that the reader had $>50 \%$ confidence in marker visualization (noted as "high confidence"). Non-visualization of marker meant that the reader had $<50 \%$ confidence in marker visualization (noted as "low confidence"). Location of the marker (cortex or outside of the cortex) and LN morphology (normal versus abnormal) was also recorded. Criteria for abnormal lymph node morphology included focal or diffuse cortical thickness of greater than $3 \mathrm{~mm}$ or replacement of the normal fatty hilum. In addition, subset analysis for HydroMARK marker appearance on T2-WO sequence was recorded as dark or bright.

Date of marker placement, date of MRI, type of marker, and date of surgery were obtained from electronic medical records. It was also noted whether the MRI was before or after NAT. Results were correlated with both clinical history of marker placement and marker retrieval at surgical pathology.

Inter-rater agreement among the readers was calculated using the intraclass correlation co-efficient (ICC). Based on Koo and Li [15], the following scale was used to interpret the intraclass coefficient: $<0.40=$ poor; $0.40-0.59=$ fair; $0.60-0.74=$ good; $0.75-1.00=$ excellent [15]. Logistic regression was performed for predicting marker visualization according to marker type, LN morphology, location of marker, and change in HydroMARK marker brightness on T2-WO images with time. Odds ratio and confidence intervals were evaluated for each subset analysis. All statistical analyses were performed using the statistical toolbox for JMP 14 (SAS Institute Inc., Cary, North Carolina 27513, USA), and $p$ 
values lower than 0.05 were considered statistically significant.

\section{Results}

A total of 80 patients had a unilateral axillary LN marker placement during the 29 -month period. Only 54 patients had an MRI after marker placement and were used in the analysis. The time interval between marker placement and MRI examination ranged from 0 to 366 days (mean 103 days; standard deviation 81 days). All the 54 patients had either pre-NAT or post-NAT MRI available for evaluation, except for one patient for whom both the pre-NAT and post-NAT MRI was available. Hence, a total of 55 MRIs were evaluated, 22 pre-NAT and 33 post-NAT. In the patient who had both pre and postNAT MRI available, these were analyzed as separate studies with the readers blinded to the information that the study was from the same patient. In the post-NAT patients, the first MRI post-initiation of NAT was evaluated.

Of 55 patients, 27 went on to axillary LN dissection. Thirty-one patients underwent sentinel LN biopsy. Of those, 25 had no positive LNs, 4 had one positive LN, and 2 had two positive LNs. Three patients with positive sentinel LN biopsy went on to complete axillary LN dissection. A total of 27 patients underwent axillary LN dissection. A range of $0-7$ positive $L N s$ were identified at final surgical pathology in patients who underwent axillary LN dissection.

\section{Marker visualization by marker type}

Of 55 markers, 34 were 18 gauge HydroMARK (Mammotome, Cincinnati, OH, USA), 10 were Tumark Professional (Hologic, Bedford, MA, USA), and 11 were "other" markers including Bard ${ }^{\circ}$ wing-shaped marker ( $n$ $=2)$, Bard ${ }^{\circ}$ ring-shaped marker $(n=2)$, Bard ${ }^{\circ}$ ribbonshaped marker $(n=2)$, Bard ${ }^{\circ}$ M-shaped marker $(n=1)$, Senomark ${ }^{\circ}$ coil-shaped marker $(n=1)$, Mammotome barrel-shaped marker $(n=1)$, Hologic ${ }^{\circ}$ stoplight-shaped marker $(n=1)$, and MammoMark ${ }^{\circ} \mathrm{U}$-shaped marker ( $n$ $=1$ ). An example of MRI imaging of U-shaped marker is provided in Fig. 1. Calculation of odds ratios was performed on all sequences/images according to marker type. The HydroMARK marker demonstrated a $3 \times$ higher likelihood of being visualized compared to the Tumark ( $p=0.003)$. "Other" markers had a $3.3 \times$ more likelihood of being visualized than Tumark $(p=0.040)$. The odds ratios for the visualization of various markers are presented in Table 1. A representative case with a Tumark marker is presented in Fig. 2.

\section{Marker visualization by type of sequence/image}

Of the 55 cases, marker visualization with high confidence among all readers was noted in 28 cases on T2-
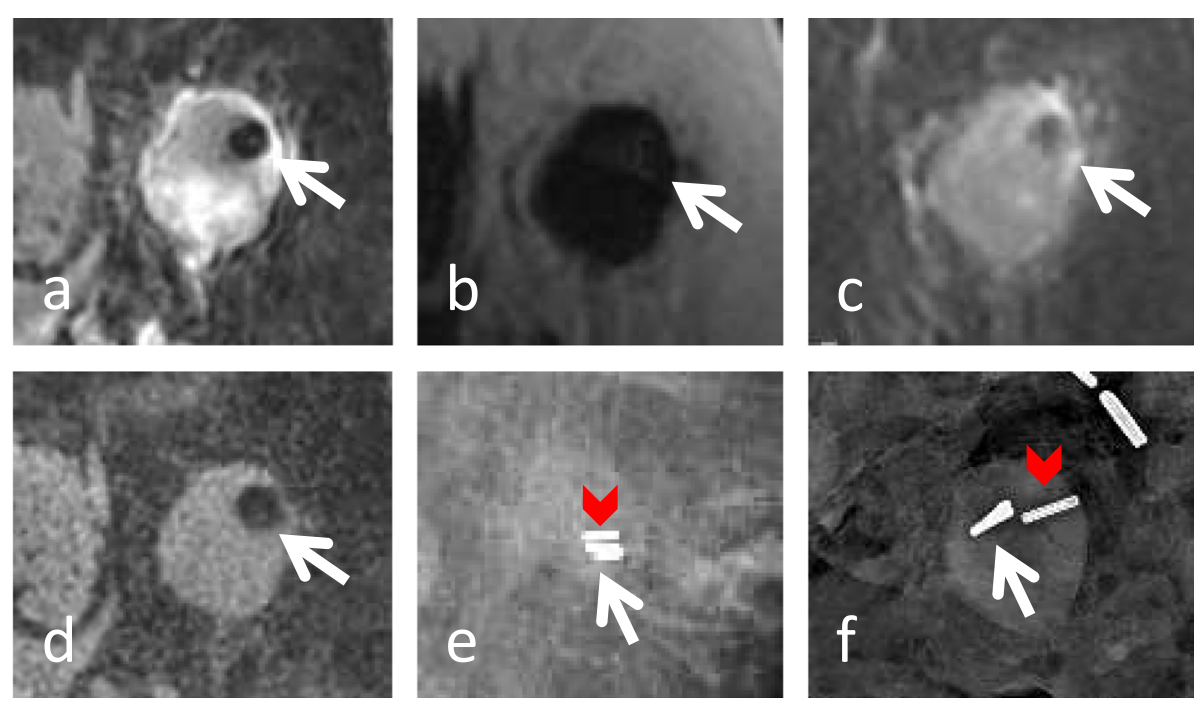

Fig. 1 A 55-year-old female with left breast multifocal invasive ductal carcinoma and biopsy proven metastatic axillary lymphadenopathy. A MammoMark ${ }^{\circledast}$ (Mammotome, Cincinnati, $\mathrm{OH}$ ) U-shaped marker was placed after biopsy of an abnormally enlarged axillary lymph node. Initial staging magnetic resonance imaging performed 1 week after marker placement demonstrates susceptibility artifact from the marker within an abnormally enlarged lymph node on various sequences. a On the T2-weighted water-only image, the marker is dark and seen with high confidence. $\mathbf{b}$ On the T2-weighted fat-only image, the marker is seen with low confidence (arrow). c On the first contrast-enhanced fatsuppressed three-dimensional T1-weighted spoiled gradient-recalled image, the marker is dark (arrow) and seen with high confidence. $\mathbf{d}$ On the unenhanced fat-suppressed three-dimensional T1-weighted spoiled gradient-recalled image, the marker is dark (arrow) and seen with high confidence. e Post-seed localization mammogram demonstrates the marker (arrow) adjacent to a radioactive seed (arrowhead/chevron). $\mathbf{f}$ Surgical specimen shows marker (arrow) and radioactive seed (arrowhead/chevron) within the metastatic lymph node 
Table 1 Odds ratios for the visualization of various marker types with high confidence

\begin{tabular}{lcccc}
\hline Marker type 1/marker type 2 & Odds ratio & $p$ value & Lower limit of confidence interval & Upper limit of confidence interval \\
\hline Tumark/HydroMARK & 0.3 & $0.030^{*}$ & 0.120 & 0.896 \\
Other markers/HydroMARK & 1.1 & 0.857 & 0.514 & 2.226 \\
Other markers/Tumark & 3.3 & $0.040^{*}$ & 1.054 & 10.129 \\
HydroMARK/Tumark & 3.1 & $0.030^{*}$ & 1.116 & 8.352 \\
HydroMARK/Other markers & 0.9 & 0.857 & 0.449 & 1.945 \\
Tumark/Other markers & 0.3 & $0.040^{*}$ & 0.099 & 0.949 \\
\hline
\end{tabular}

*Statistically significant

WO images, in 22 cases on the first contrast-enhanced SPGR images, in 9 cases on unenhanced SPGR images, and in only 1 case on T2-FO images. Evaluation of odds ratios performed for all the markers demonstrated the following: a marker was $3.3 \times$ more likely to be seen on the first contrastenhanced SPGR sequence compared to the unenhanced SPGR sequence $(p=0.009)$. A marker was $5.2 \times$ more likely to be seen on the T2-WO sequence compared to the unenhanced SPGR sequence $(p<0.001)$ and $1.6 \times$ more likely to be seen on T2-WO sequence compared to the first contrast-enhanced SPGR sequence $(p=0.252)$. The odds ratios of the various sequences are summarized in Table 2.

A subset analysis was performed for visualization of the HydroMARK marker, since this was the majority of marker type $(n=34)$. Marker visualization with high confidence among all readers was noted in 17 cases on T2-WO images, in 10 cases on the first contrast-enhanced SPGR images, in 3 cases on unenhanced SPGR images, and zero cases on T2-FO images. Odds ratios for visualization of the HydroMARK marker were calculated. First contrast-enhanced SPGR sequence was $6.4 \times$ more likely to show the HydroMARK marker compared to pre unenhanced SPGR sequence $(p=0.003)$. The HydroMARK marker was $11.7 \times$ more likely to be seen on the T2-WO sequence compared to the unenhanced SPGR sequence $(p<$ 0.001 ) and $1.8 \times$ more likely to be seen on the T2WO sequence compared to the first contrast-
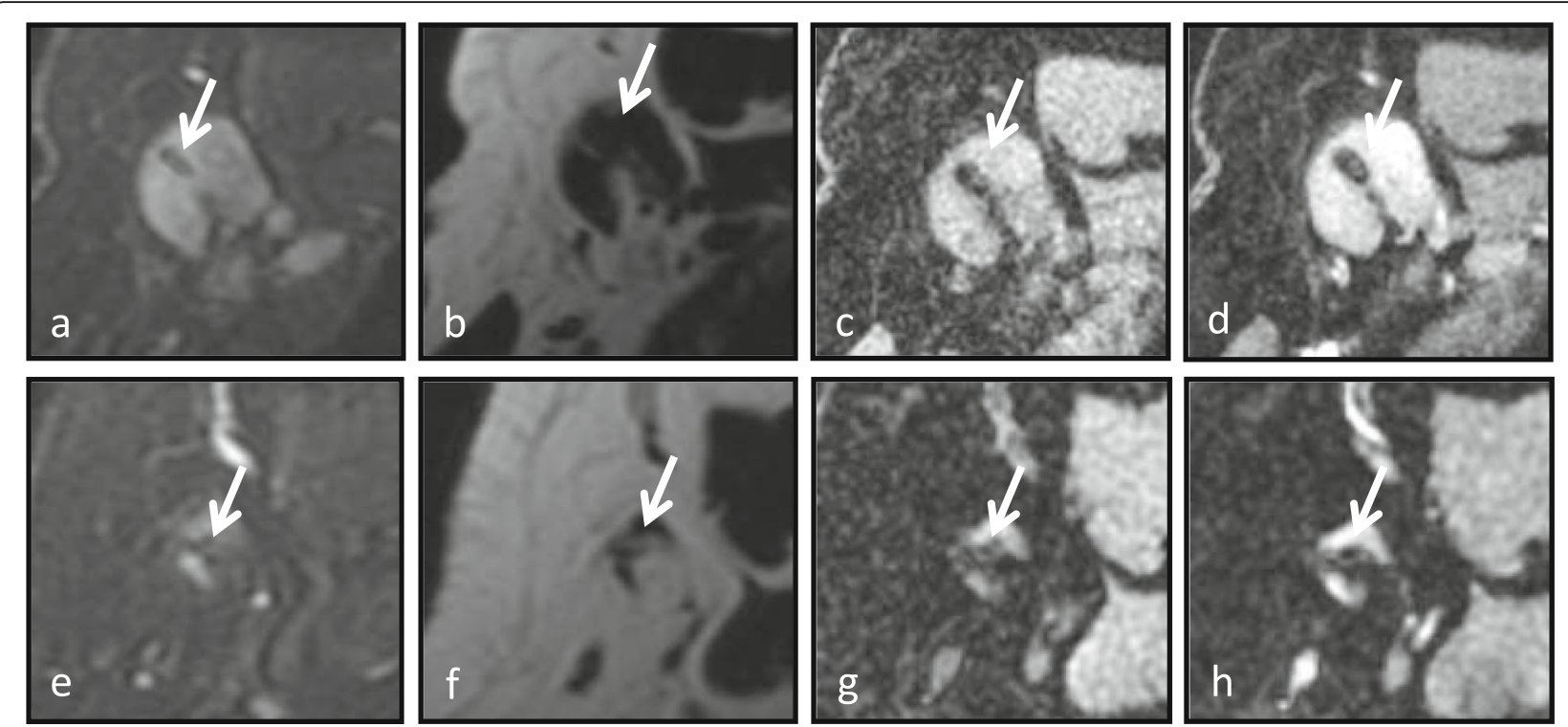

Fig. 2 A 49-year-old female with invasive lobular carcinoma of the right breast with biopsy proven right axillary metastatic lymphadenopathy. A Tumark $x$-shaped marker was placed at site of axillary lymph node biopsy. a-d Initial staging magnetic resonance imaging (MRI) 2 weeks after lymph node marker placement. The marker is seen with high confidence on the T2-weighted water-only fast spin-echo image (a), the unenhanced fat-suppressed three-dimensional T1-weighted spoiled gradient-recalled image (c), and the first contrast-enhanced fat-suppressed three-dimensional T1-weighted spoiled gradient-recalled image (d), while it is seen with low confidence on the T2-weigheted fat-only fast spinecho image (b). In the follow-up of neoadjuvant therapy, MRI was performed 6 months after biopsy marker placement. The marker is seen with low confidence on all images: T2-weighted water-only fast spin-echo image (e), T2-weighted fat-only fast spin-echo image (f), unenhanced fatsuppressed three-dimensional T1-weighted spoiled gradient-recalled image (g), and first contrast-enhanced fat-suppressed three-dimensional T1weighted spoiled gradient-recalled image (h), with poor visualization attributed to the decreased size of lymph node cortical thickness 
Table $\mathbf{2}$ Odds ratios for the visualization of markers on the different images evaluated

\begin{tabular}{lrrrr}
\hline Sequence 1/sequence 2 & Odds ratio & $p$ value & Lower limit of confidence interval & Lower limit of confidence interval \\
\hline T2-FO/CE-SPGR & 0.0 & $0.001^{*}$ & 0.004 & 0.216 \\
T2-FO/SPGR & 0.1 & $0.027^{*}$ & 0.011 & 0.759 \\
CE-SPGR/SPGR & 3.3 & $0.009^{*}$ & 1.360 & 8.168 \\
T2-FO/T2-WO & 0.0 & $<0.001^{*}$ & 0.002 & 0.138 \\
CE-SPGR/T2-WO & 0.6 & 0.252 & 0.302 & 1.368 \\
SPGR/T2-WO & 0.2 & $<0.001^{*}$ & 0.079 & 0.470 \\
CE-SPGR/T2-FO & 36 & $0.001^{*}$ & 4.633 & 279.721 \\
SPGR/T2-FO & 10.8 & $0.027^{*}$ & 1.318 & 88.506 \\
SPGR/CE-SPGR & 0.3 & $0.009^{*}$ & 0.122 & 0.735 \\
T2-WO/T2-FO & 56 & $<0.001^{*}$ & 7.228 & 433.896 \\
T2-WO/CE-SPGR & 1.6 & 0.252 & 0.731 & 3.3106 \\
T2-WO/SPGR & 5.2 & $<0.001^{*}$ & 2.130 & 12.624 \\
\hline
\end{tabular}

CE-SPGR First contrast-enhanced fat-suppressed three-dimensional T1-weighted spoiled gradient-recalled images, SPGR Unenhanced fat-suppressed threedimensional T1-weighted spoiled gradient-recalled images, T2-FO T2-weighted fat-only fast-spin-echo images, T2-WO T2-weighted water-only fast spin-echo images. *Statistically significant

enhanced SPGR sequence, but these results were not statistically significant $(p=0.225)$. The HydroMARK marker was not seen with high confidence on the T2FO sequence. A representative case with a HydroMARK marker is shown in Fig. 3.

\section{Marker appearance on T2-WO images}

Overall, of the 43 markers seen with high confidence on the T2-WO images, 24 were hyperintense and 19 were hypointense. Of the 28 HydroMARK markers seen with high confidence on the T2-WO images, 21 were
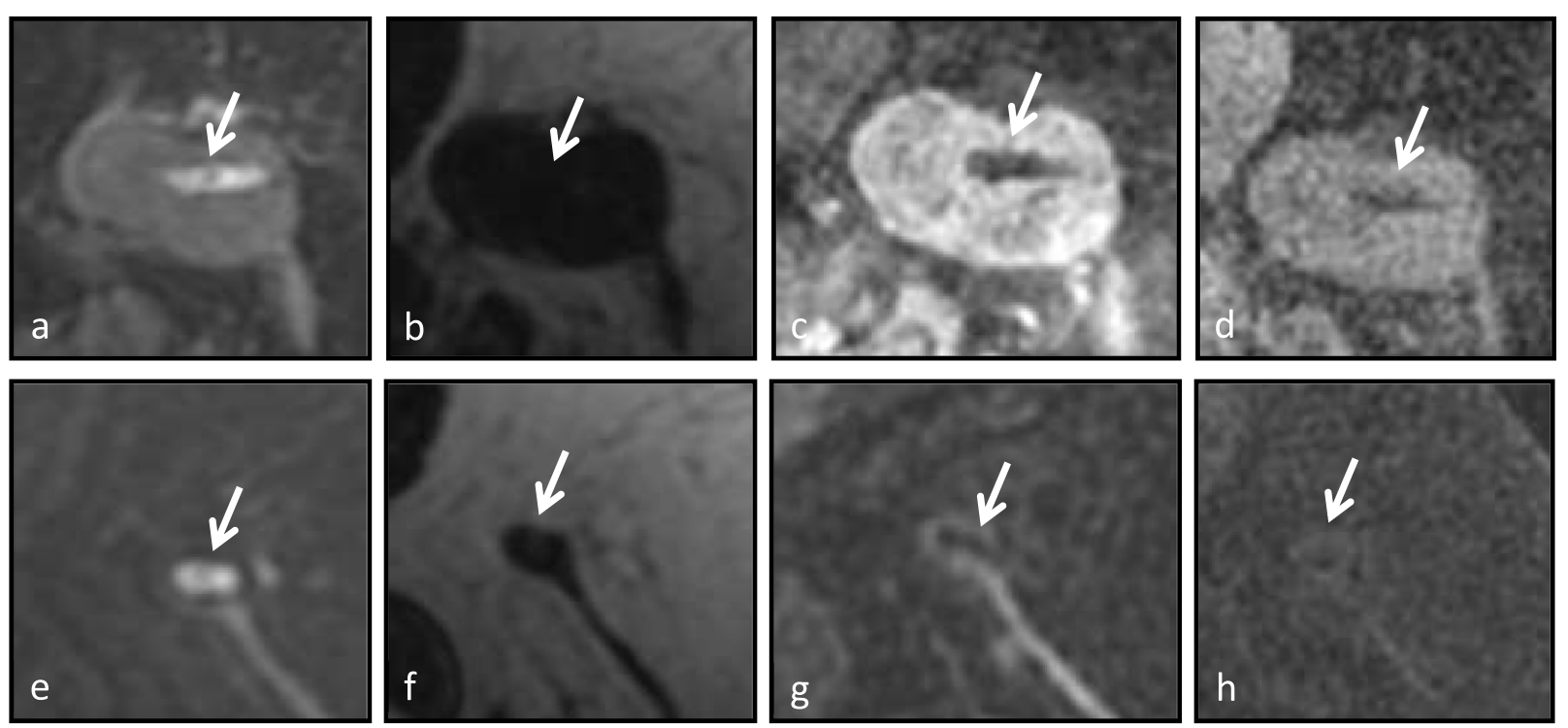

Fig. 3 A 59-year-old female with newly diagnosed left breast invasive ductal carcinoma grade 3 with known metastatic left axillary lymphadenopathy. a-d Initial staging magnetic resonance imaging (MRI) performed 21 days after HydroMARK placement. The marker (arrow) is seen with high confidence on the T2-weigheted water-only fast spin-echo image (a), the unenhanced fat-suppressed three-dimensional T1weighted spoiled gradient-recalled image (c), and the first contrast-enhanced fat-suppressed three-dimensional T1-weighted spoiled gradientrecalled image (d), while it is seen with low confidence on the T2-weigheted fat-only fast spin-echo image (b). e-h NAT follow-up MRI performed approximately 6 months after marker placement shows decreased size of lymph node. After NAT, the marker (arrow) is seen with high confidence on the T2-weigheted water-only fast spin-echo image (e) and the first contrast-enhanced fat-suppressed three-dimensional T1weighted spoiled gradient-recalled image $(\mathbf{h})$ sequences, while it is seen with low confidence visibility on the T2-weigheted fat-only fast spinecho image (f) and the unenhanced fat-suppressed three-dimensional T1-weighted spoiled gradient-recalled image (g). Note, the decreased T2 brightness of the marker gel content 6 months after placement (e) compared to the initial MRI after placement (a) 


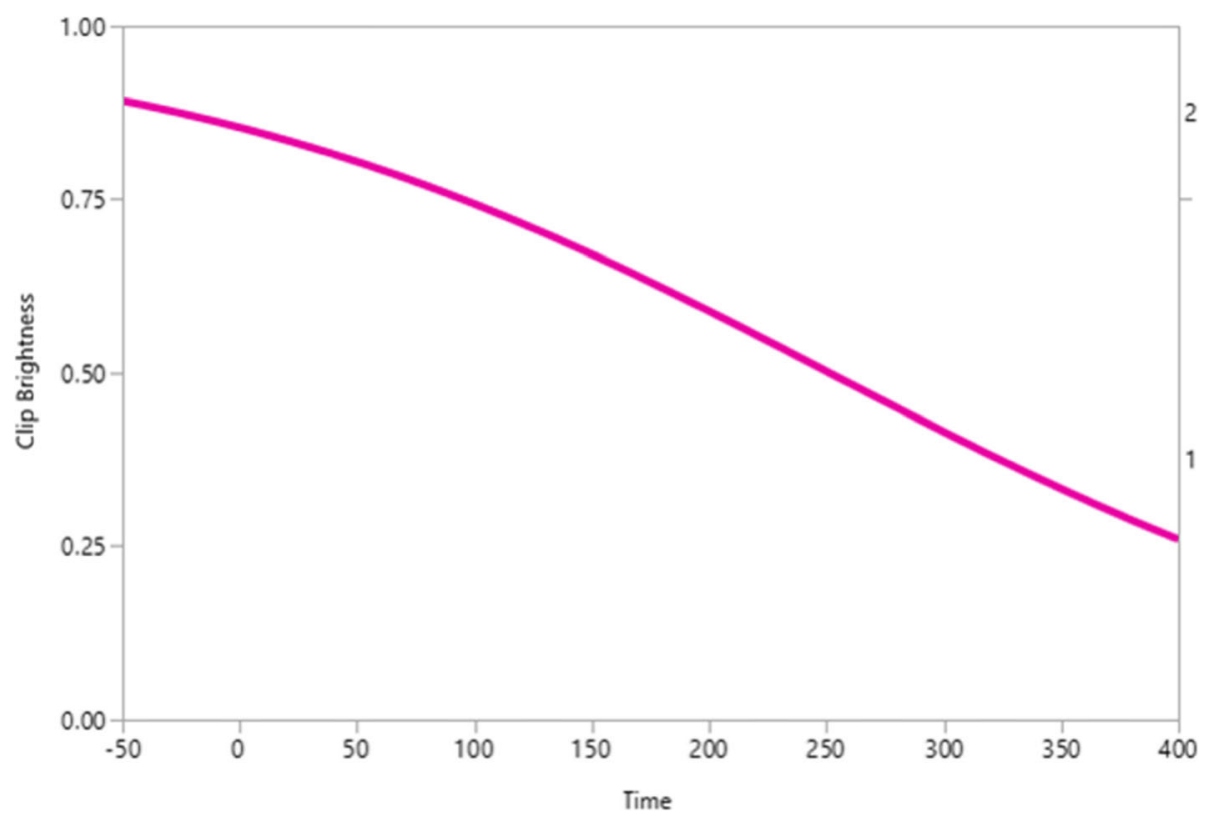

Fig. 4 Plot for marker brightness versus time (days). Data are referred to HydroMARK marker on T2-weighted water-only fast spin-echo images

hyperintense and 7 were hypointense. Of the 6 Tumark markers seen with high confidence on the T2-WO images, 2 were hyperintense and 4 were hypointense. Of the 9 "other" markers seen with high confidence on the T2-WO images, 1 was hyperintense and 8 were hypointense. HydroMARK marker brightness on the T2-WO images specifically decreased with time. After 250 days, confidence in HydroMARK marker brightness was less than $50 \%(p<0.001)$. These findings are presented in Fig. 4.

\section{Marker location}

Of the total 55 markers, 40 were noted to be within the cortex, while the remaining 15 were noted to be outside the cortex (within hilum or surrounding tissues). A marker was more $1.2 \times$ more likely to be visualized if placed in the cortex versus out of the cortex, but the results were not statistically significant $(p=0.625)$.

\section{LN morphology}

Of the 55 lymph nodes with marker placement, 26 were categorized as normal morphology, and 29 lymph nodes were categorized as abnormal in our study. A marker was $8.4 \times$ more likely to be visualized if the node was abnormal in size/morphology $(p<0.001)$. A representative case from a marker belonging to the "other" category placed within an abnormal LN is shown in Fig. 1.

\section{Inter-reader agreement}

Inter-rater agreement was excellent for T2-WO images (single measure ICC 0.76), excellent for first contrastenhanced SPGR images (single measure ICC 0.76), good for unenhanced SPGR images (single measure ICC 0.60), and poor for the T2-FO images (single measure ICC $0.24)$.

\section{Discussion}

Although localization of axillary LN markers is typically not performed with MRI, identification of pathologic axillary LNs on MRI is very important in the staging and treatment of breast cancer. Many LNs decrease in size after NAT making it harder to identify which $\mathrm{LN}$ was metastatic, and in such cases, the marker can be used as a point of reference to monitor response to NAT. There is not much data on MRI sequences that are best to identify biopsy markers within axillary LNs. Multiple studies, however, have evaluated MRI characteristics that help identify pathologic LNs [16-22]. A systematic review demonstrated that a protocol with unenhanced T1- and T2weighted with ultra-small iron particles-enhanced $\mathrm{T} 2$ * weighted sequences and a dedicated axillary protocol was most promising in identifying metastatic axillary LNs [23]. The negative predictive value of such a protocol was found to be similar to that of sentinel LN biopsy. It was also suggested that a dedicated axillary protocol, for example, one that uses a radiofrequency coil placed on the axilla, was better than a more standard protocol covering the breast and axilla in the same field of view [23].

In our study, we looked at various factors affecting marker visibility in patients receiving or not receiving NAT. The T2-WO and contrast-enhanced SPGR images overall demonstrated the best likelihood of marker visualization. It was also noted that contrast-enhanced 
images were better to identify markers compared to unenhanced images, and that markers placed within morphologically abnormal LNs were better visualized. These findings are explained by the dark signal void (susceptibility artifact) caused by the biopsy marker. The dark susceptibility artifact makes the marker easy to identify within the hyperintense LN cortex on T2-WO, and within the homogenous enhancement of the LN cortex on postcontrast sequences when the marker was placed in the cortex, especially when the cortex is morphologically enlarged. When markers are placed in the hilum, the signal void from the marker can sometimes be difficult to separate from the surrounding suppressed fat which also appears hypointense on MRI.

In our study, HydroMARK markers were significantly better visualized on MRI compared to Tumark markers, which may be a consideration during marker placement within metastatic axillary LNs. In axillary LNs containing the HydroMARK marker, the gel within the marker was easily identifiable on T2-WO images due to its hyperintense signal from the water content. Our study also demonstrated that the brightness within this marker decreased with time as expected, and after 250 days, confidence in identifying marker brightness was less than $50 \%$ for the HydroMARK marker. This is secondary to the gel content within the HydroMARK marker gradually being resorbed with time [13].

Once the marker is identified within the metastatic LN, even if the LN decreases in size after NAT, the anatomical landmarks surrounding the metastatic node may be used to assess response to NAT on MRI. For example, it can be noted if the marker is within the high axilla or low axilla, and other adjacent LNs and surrounding vessels can be used as landmarks to help determine the marked node on subsequent MRIs after NAT, even if the marker is less conspicuous with time. We found the T2-FO images to be very poor in identification of the biopsy marker for all types, as a lot of artifact was noted at the fat/water interfaces, making it difficult to separate from the biopsy marker. There was no statistically significant difference between markers placed in the cortex and outside of the cortex in our study but abnormal LN morphology did make it easier to identify the marker.

The inter-reader agreement between the three readers was overall good or excellent in our study (with the only exception of T2-FO images), adding more confidence into our results.

Limitations of this study include that this is a retrospective review performed at a single institution with a relatively small sample size. Larger studies would help increase the confidence in our results, although the number of our patients was enough to reach statistical significance. Additionally, it is possible that artifact could have been mistaken for a marker within the axillary nodes. However, this is why the confidence scale was set to less than $50 \%$ for non-visualization of marker; therefore, the authors had to be $>50 \%$ confident that this was indeed a marker and not an artifact. Our study is also limited by the number of various markers types that were included, as we had more HydroMARK markers compared to Tumark and markers in the "other" category. Moreover, images were reviewed in the order that they had been acquired (T2-WO, T2-FO, unenhanced SPGR, and contrastenhanced SPGR). This may have introduced some bias, as knowledge from one sequence may have influenced marker evaluation on the next sequence from the same patient. However, once a sequence had been assessed, the readers were not allowed to change their results or go back to a previously evaluated sequence to compare it to the next. Another limitation is that only one patient within this study had both before- and after-NAT MRI, and both MRIs were used independently for analysis, with readers being blinded to the information that it was the same patient. It would be interesting to evaluate the appearance on pre- and post-NAT MRIs on subsequent studies. Finally, we only evaluated sequences that are specific to our General Electric MRI scanner and protocol. Other sequences that may be used at other institutions such as STIR, in phase non-fat saturated, and spectrally fat-suppressed T2-weighted sequences were not evaluated.

In conclusion, our study showed that axillary $\mathrm{LN}$ markers are best seen on T2-WO and contrast-enhanced SPGR images, and the HydroMARK was better visualized compared to Tumark. In addition, we found that markers were easier to identify when placed in abnormal LNs and that the visibility of HydroMARK marker was reduced with time, likely due to its gel content being resorbed.

\section{Abbreviations \\ ICC: Intraclass correlation coefficient; LN: Lymph node; MRI: Magnetic resonance imaging; NAT: Neoadjuvant therapy; SPGR: Spoiled gradient- recalled; T2-FO: T2-weighted fat-only fast-spin-echo; T2-WO: T2-weighted water-only fast spin-echo}

\section{Authors' contributions}

$N S, K A, A B, K G$, and $S Z$ were all involved in data mining and evaluation of cases. NS, $A B$, and $K G$ were involved in the inter-reader study. NS did the preliminary draft of the manuscript and $A B$ and $K G$ were involved revising and supervision. The authors read and approved the final manuscript.

\section{Funding}

No funding was obtained for this study

\section{Availability of data and materials \\ The datasets used and/or analyzed during the current study are available from the corresponding author on reasonable request.}

Ethics approval and consent to participate

Retrospective study approved by the corresponding author's Institutional Review Board Mayo Clinic Rochester, MN, October 17, 2016, ID 16-008036. Reviewer approved waiver of the requirement to obtain informed consent in accordance with 45 CFR 46.116.

Consent for publication

See the "Ethics approval and consent to participate" section. 


\section{Competing interests}

The authors declare that they have no competing interests.

\section{Author details}

'NYU Langone, 765 Stewart Ave, Garden City, NY 11530, USA. ²Department of Radiology, Mayo Clinic, 200 1st street SW, Rochester, MN 55905, USA. ${ }^{3}$ St Vincent Healthcare, 2900 12th Ave N, Billings, MT 59101, USA.

Received: 19 December 2019 Accepted: 9 April 2020

Published online: 12 June 2020

\section{References}

1. Gampenrieder SP, Peer A, Weismann C et al (2019) Radiologic complete response ( $\mathrm{rCR}$ ) in contrast-enhanced magnetic resonance imaging (CE-MRI) after neoadjuvant chemotherapy for early breast cancer predicts recurrencefree survival but not pathologic complete response (pCR). Breast Cancer Res 21:19. https://doi.org/10.1186/s13058-018-1091-y

2. Rauch GM, Adrada BE, Kuerer HM, van la Parra RFD, Leung JWT, Yang WT (2016) Multimodality imaging for evaluating response to neoadjuvant chemotherapy in breast cancer. AJR Am J Roentgenol 208:290-299. https:// doi.org/10.2214/AJR.16.17223

3. Jatoi I (2010) Surgical considerations in the management of primary invasive breast cancer. In: Jatoi I, Kaufmann M (eds) Management of breast diseases. Springer, Berlin Heidelberg, Berlin, Heidelberg, pp 227-241. https:// doi.org/10.1007/978-3-540-69743-5_13

4. Jatoi I, Benson JR (2018) Surgical management of the axilla in early breast cancer. Curr Probl Surg 55:47-65. https://doi.org/10.1067/j.cpsurg.2018.01. 003

5. Peeters MV (2009) Axillary staging: new approaches and treatment of minimal disease. Breast Cancer Res 11:S6. https://doi.org/10.1186/bcr2267

6. Ecanow JS, Abe H, Newstead GM, Ecanow DB, Jeske JM (2013) Axillary staging of breast cancer: what the radiologist should know. Radiographics 33:1589-1612. https://doi.org/10.1148/rg.336125060

7. Kalli S, Semine A, Cohen S, Naber SP, Makim SS, Bahl M (2018) American joint committee on cancer's staging system for breast cancer, eighth edition: what the radiologist needs to know. Radiographics 38:1921-1933. https://doi.org/10.1148/rg.2018180056

8. Ha SM, Cha JH, Kim HH, Shin HJ, Chae EY, Choi WJ (2017) Diagnostic performance of breast ultrasonography and mri in the prediction of lymph node status after neoadjuvant chemotherapy for breast cancer. Acta Radiol 58:1198-1205. https://doi.org/10.1177/0284185117690421

9. Javid S, Segara D, Lotfi P, Raza S, Golshan M (2010) Can breast mri predict axillary lymph node metastasis in women undergoing neoadjuvant chemotherapy. Ann Surg Oncol 17:1841-1846. https://doi.org/10.1245/ s10434-010-0934-2

10. Hieken TJ, Boughey JC, Jones KN, Shah SS, Glazebrook KN (2013) Imaging response and residual metastatic axillary lymph node disease after neoadjuvant chemotherapy for primary breast cancer. Ann Surg Oncol 20: 3199-3204. https://doi.org/10.1245/s10434-013-3118-z

11. Caudle AS, Yang WT, Krishnamurthy $S$ et al (2016) Improved axillary evaluation following neoadjuvant therapy for patients with node-positive breast cancer using selective evaluation of clipped nodes: implementation of targeted axillary dissection. J Clin Oncol 34:1072-1078. https://doi.org/10. 1200/JCO.2015.64.0094

12. Kim EY, Byon WS, Lee KH et al (2018) Feasibility of preoperative axillary lymph node marking with a clip in breast cancer patients before neoadjuvant chemotherapy: a preliminary study. World J Surg 42:582-589. https://doi.org/10.1007/s00268-017-4171-8

13. Mammotome (2019, November) Hydromark breast biopsy marker. https:// www2.mammotome.com/hydromark-breast-marker?gclid= CjOKCQjw09HzBRDrARIsAG60GP8E MurQxbnpTQYgOANPH9N7WBvgnmTpo3fOALY1aFh6lGItiuAaQaAnsGEALw_wcB. Accessed 15 Dec 2019

14. Hologic (2020) Breast biopsy markers - tumark biopsy site markers. https:// www.hologic.com/hologic-products/breast-skeletal/breast-biopsy-markers. Accessed 15 Dec 2019

15. Koo TK, Li MY (2016) A guideline of selecting and reporting intraclass correlation coefficients for reliability research. J Chiropr Med 15:155-163. https://doi.org/10.1016/j.jcm.2016.02.012

16. Schipper R-J, Paiman M-L, Beets-Tan RGH et al (2014) Diagnostic performance of dedicated axillary t2- and diffusion-weighted mr imaging for nodal staging in breast cancer. Radiology 275:345-355. https://doi.org/ 10.1148/radiol.14141167

17. Scaranelo AM, Eiada R, Jacks LM, Kulkarni SR, Crystal P (2012) Accuracy of unenhanced $\mathrm{mr}$ imaging in the detection of axillary lymph node metastasis: study of reproducibility and reliability. Radiology 262:425-434. https://doi. org/10.1148/radiol.11110639

18. Memarsadeghi M, Riedl CC, Kaneider A et al (2006) Axillary lymph node metastases in patients with breast carcinomas: assessment with nonenhanced versus uspio-enhanced mr imaging. Radiology 241:367-377. https://doi.org/10.1148/radiol.2412050693

19. Stadnik TW, Everaert H, Makkat S, Sacré R, Lamote J, Bourgain C (2006) Breast imaging. Preoperative breast cancer staging: comparison of uspioenhanced $\mathrm{mr}$ imaging and 18f-fluorodeoxyglucose (fdc) positron emission tomography (pet) imaging for axillary lymph node staging -initial findings. Eur Radiol 16:2153-2160. https://doi.org/10.1007/s00330-006-0276-4

20. Luciani A, Dao TH, Lapeyre M et al (2004) Simultaneous bilateral breast and high-resolution axillary mri of patients with breast cancer: preliminary results. AJR Am J Roentgenol 182:1059-1067. https://doi.org/10.2214/ajr.182. 4.1821059

21. Kvistad KA, Rydland J, Smethurst HB, Lundgren S, Fjøsne HE, Haraldseth O (2000) Axillary lymph node metastases in breast cancer: preoperative detection with dynamic contrast-enhanced mri. Eur Radiol 10:1464-1471. https://doi.org/10.1007/s003300000370

22. Jung $\mathrm{N}$, Kim HJ, Jung JH et al (2019) Restaging the axilla after neo-adjuvant chemotherapy for breast cancer: predictive factors for residual metastatic lymph node disease with negative imaging findings. J Breast Imaging 25: 196-201. https://doi.org/10.1111/tbj.13192

23. Kuijs VJL, Moossdorff M, Schipper RJ et al (2015) The role of mri in axillary lymph node imaging in breast cancer patients: a systematic review. Insights Imaging 6:203-215. https://doi.org/10.1007/s13244-015-0404-2

\section{Publisher's Note}

Springer Nature remains neutral with regard to jurisdictional claims in published maps and institutional affiliations.

\section{Submit your manuscript to a SpringerOpen ${ }^{\circ}$ journal and benefit from:}

- Convenient online submission

- Rigorous peer review

- Open access: articles freely available online

High visibility within the field

- Retaining the copyright to your article

Submit your next manuscript at $\boldsymbol{\nabla}$ springeropen.com 